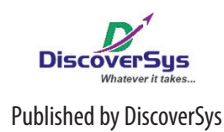

Published by DiscoverSys

\title{
Consideration of Including Male Circumcision in the Indonesian HIV Prevention Strategy
}

\author{
I Nyoman Sutarsa*
}

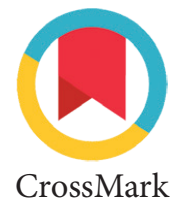

Department of Community and Preventive Medicine Faculty of Medicine Udayana University
*Correspondence to: I Nyoman Sutarsa, Department of Community and Preventive Medicine Faculty of Medicine Udayana University sutarsa84@gmailcom

\section{INTRODUCTION}

HIV/AIDS is an emerging threat to population health. Globally, 33.4 million people were estimated to be living with HIV in 2008 including 2.1 million children (UNAIDS, 2009; UNAIDS, 2011). The total number of new cases was estimated to be 2.7 million people (including 430,000 children) and HIV/AIDS related death was estimated to be 2.0 million in 2008 (UNAIDS, 2009). Sustainable prevention measures followed by care, support and treatment program is vital to reduce the incidence and prevalence of HIV/AIDS.

Currently available HIV/AIDS estimation procedures tend to be overestimated, resulting in misclassification of HIV epidemic levels. This is clearly illustrated in Cambodia, where antenatal survey found the prevalence of HIV in general population was $2.6 \%$ whereas the preliminary finding from population-based estimates is less than $1 \%$ (Wilson, 2006). Generalised epidemic is defined as HIV prevalence in the general population higher than 1\% (UNICEF, 2011). Using the result from antenatal survey as an indicator, Cambodia is classified as 'generalised epidemic'. Using the result from population based estimates, Cambodia is classified as 'concentrated epidemic'. The prevention strategies within generalised and concentrated epidemics are vastly different. In generalised epidemic, normative changes and reducing sexual partners in general population is critical followed by reinforcing condom use. In contrast, promoting condom use followed by reducing commercial sex clients is critical in concentrated epidemic (Wilson, 2006). Several behaviour changes have been shown to significantly reduced HIV transmission. Examples of these include partner reduction among adults and increasing condom use in Uganda and Zimbabwe; deferred sexual inception among youths in Kenya and Zimbabwe; and increasing condom use among sex workers followed by reduction of commercial sex clients in Thailand, Cambodia and South India (Wilson, 2006).

Male circumcision correlates to HIV transmission reduction in highly epidemic areas. Systematic Review (SR) on 'male circumcision for prevention of heterosexual acquisition of $\mathrm{HIV}$ in men' conducted by Siegfried et al. (2009) was generated from three large sample size Randomised
Controlled Trials (RCTs) in South Africa, Kenya and Uganda. The authors concluded there is strong evidence that medical male circumcision reduces the acquisition of HIV by heterosexual men by between 38-66\%. They also report low incidence of adverse effects after 24 months follow up, indicating this is a safe procedure. They suggest that inclusion of male circumcision into current HIV prevention measure guidelines is warranted, however further research to assess feasibility, cost effectiveness and desirability is required prior to implementation. The authors strongly believe that male circumcision will prevent men in the general population from acquiring HIV from heterosexual intercourse (Siegfried et al., 2009)

This finding, however, is not transferable into all HIV epidemic areas. Critical considerations of health system, level of HIV epidemic and cultural acceptability must be taken into account before integrating adult male circumcision as a part of national or local prevention guidelines.

\section{DISCUSSION}

In 2007, WHO and UNAIDS delivered a press release advocating the inclusion of male circumcision into current HIV prevention guidelines in countries with low circumcision rates and generalised epidemic (WHO \& UNAIDS, 2007). The SR conducted by Siegfried et al. (2009) supported this and recommended the implementation of male circumcision if feasible and culturally acceptable. Siegfried et al. (2009) also suggest that male circumcision should be complemented by other prevention measures (eg. promoting consistent condom use and sexual partner reduction). ${ }^{5}$ This strong evidence however is not practically transferable into Indonesian context due to acceptability and feasibility issues. These include (1) the HIV/AIDS epidemic situation in Indonesia still largely focussing on the centrality of female sex workers; (2) social reluctance in some non-Islamic local areas due to circumcision being strongly associated with Muslims and circumcision being mostly viewed as a traditional practice (not correlated with health benefits); and (3) the health system not being ready to cover potential demand for circumcisions. 


\section{HIV/AIDS epidemic and the centrality of sex workers}

Africa is the epicentre of the global HIV epidemic. Wilson argues that this is a continental and global exception which is unlikely to occur elsewhere. ${ }^{3}$ Further analysis of Africa's epidemic shows four distinct clusters: (1) Southern Africa (15-35\%), classified as highly generalised epidemic, (2) Eastern Africa (2-7\%), (3) West Africa (1-5\%) and (4) North Africa (0-0.1\%). The later three areas are classified as less generalised or concentrated epidemics (Wilson, 2006). Looking at the HIV prevalence alone in deciding male circumcision implementation may lead to 'false' prevention strategies. Even though national prevalence of HIV/AIDS in Indonesia is low (0.2\%), there are several local areas that are highly concentrated and generalised epidemics such as Papua, West Papua and Bali (Ministry of Health of Indonesia, 2010 cited in Indonesian National AIDS Commission, 2011b).In contrast to Africa, HIV vulnerable groups (ie. commercial sex workers and clients) form a large proportion of the adult population with HIV/AIDS in Indonesia. This produces localised prevalence estimates more than $1 \%$ in highly concentrated and generalised epidemic areas without significant transmission in the general population (Wilson, 2006).

In Indonesia, HIV transmission is primarily attributable to HIV vulnerable groups, therefore protecting these groups would protect the wider community (Indonesian National AIDS Commission, 2011a). The rising HIV infection among non sex worker women (increasing from 1999-2010) is generated from their male partners who visit commercial sex workers (Indonesian National AIDS Commission, 2011a). This circumstance places sex workers at the epicentre of HIV/AIDS epidemic in Indonesia. Indeed, the prevalence of HIV/AIDS in commercial sex workers in Indonesia is high, reaching $6.06 \%$ in 2010 (Indonesian National AIDS Commission, 2011a). This indicated the HIV epidemic in Indonesia is not primarily driven by sexual behaviour in the general population. In contrast, most of the countries in Africa including South Africa, Kenya and Uganda have a low prevalence of new HIV infection related to sex workers. In Kenya for instance, the prevalence of HIV in sex workers is $60 \%$ however less than $50 \%$ of new HIV infection relates to sex work. Similar feature can be drawn from South Africa where HIV prevalence among sex workers is $50 \%$, but less than $5 \%$ of new HIV infection relates to sex work (Wilson, 2006). These circumstances indicate that HIV epidemic in those areas is primarily driven by sexual behaviour in the general population.
The above discussion suggests that large scale and fundamental changes in community norms, sexual values and practices are less appropriate in the Indonesian context. Specific measures to protect sex workers and clients are far more critical for reducing HIV transmission to the wider community. Expanding the coverage of existing proven interventions could include increasing consistent condom use among sex workers and clients. In Thailand for example, $89 \%$ of sexual transmitted infections were reduced by increasing condom use in commercial sex practices (Wilson, 2006).

\section{Cultural and social reluctant}

Male circumcision in Indonesia is a common practice amongst the Islamic population. Horison (2000) highlighted that male circumcision can be part of tradition or culture in some regions and not necessarily for health purposes only (cited in Siegfried et al., 2009). Majority of the Indonesian population is Islamic, in which male circumcision is an inherited religious practice. Introducing male circumcision into the general population of Bali (which is majority Hindu) will encounter social reluctance as they perceive this measure as an instrument of 'Islamisation'. In contrast, vast majority of RCT participants in Kenya receiving male circumcision were Christian (Siegfried et al., 2009), indicating male circumcision may be easier to introduce in the majority Christian island of Papua, Indonesia.

Siegfried et al. (2009) stated that adverse effects from male circumcision can be reduced with regular ongoing monitoring from health professional. In Indonesia however, adverse effects after circumcision are far more prevalent because circumcisions are largely done as a traditional practice (not correlated with health benefits) without adequate ongoing medical monitoring. This result in low social willingness amongst non-Islamic people to circumcise their children or themselves, unless they become sick and medical professionals recommend circumcision. Social perception, for instance the false view of male circumcision as a comparable substitute to condom use in preventing HIV transmission (Bridges et al., 2011) should also be taken into consideration before male circumcision is introduced into general population. Some recent studies provide insight on possible directions for future implementation of male circumcision to non-Islamic areas of Indonesia. Dini (2010) argues that neonatal male circumcision is more socially acceptable and feasible to be implemented..$^{10}$ Brito et al. argue that promotional campaign on health benefit of male circumcision involving local community leaders 
and religious agencies should be in place to increase social acceptance. Kenneth et al. (2011) argue that engaging both parents in an educational campaign may increase interest in medical male circumcision for HIV prevention.

Some of the findings of Siegfried et al. (2009) on behavioural implications of male circumcision could potentially reduce the social acceptability. Premature resumption of sex after male circumcision can actually increase risk of HIV transmission to women during the period when the man's wound has not fully healed. In the trial groups, about $13 \%$ of women partners still acquire HIV infection (Siegfried et al., 2009). This finding potentially forms lower acceptability in patriarchy culture. Hellet et al. (2011) argues that irrespective of premature resumption of sex the overall long term benefit to woman from male circumcision is still significant-suggesting greater imperative to increase scale of male circumcision services. The fact that male circumcision has potential to increase risky sexual behaviours (by 2-8\%) via disinhibiting mechanism (Siegfried et al., 2009) may also reduce its acceptability. This is because there is a common stigma in Indonesian culture that all people with HIV/AIDS contracted it via engaging with the sex industry. Siegfried et al. claim male circumcision is effective in reducing HIV transmission by up to $66 \%$, however the trials were only conducted over 24 months. ${ }^{5}$ The long term effectiveness remains somewhat unknown. Eaton \& Seth (2009) also suggest further studies are needed to explore the importance of behavioural implications of male circumcision for HIV prevention. Circumcised men tended to form the belief that it will give absolute protection (Moses, 2011). This also correlates to low acceptability and feasibility in the broader community of male circumcision for HIV prevention measure.

\section{Health system readiness}

Siegfried et al. (2009) suggest male circumcision should be conducted by an appropriate and skilled practitioner in a sterile clinical environment. Male circumcision under surgical procedures with regular follow up monitoring during the trial period resulted in reduced levels of adverse effects. Three main common adverse effects are pain, excessive bleeding and swelling or appearance problems (Siegfried et al., 2011). This finding provides strong evidence that male circumcision can be done safely. Delivering large scale male circumcision under these specific conditions is quite challenging though. Providing trained and skilful health professionals coupled with satisfactory infrastructures to support this practice requires substantial amounts of resource.
Consideration of assumed controlled risk (ACR) and number needed to treat (NNT) are also issues. Assuming low ACR of HIV infection is $1 \%$ over two years will result in $186 \mathrm{NNT}$ and potentially prevent 5 persons per 1000 men from contracting HIV/AIDS within two years. Higher ACR as 3\% results in 62 NNT and potentially prevents 16 persons per 1000 men from contracting HIV/AIDS within two years (Siegfried et al., 2009). The higher ACR produces lower NNT and higher number of prevention. Applying this assumption in Indonesian (where prevalence is $0.2 \%$ ), will yield 930 NNT and potentially prevent 2 persons per 1000 men from contracting HIV/ AIDS within two years. This demand is extremely high for the current Indonesian health system. Moreover, the high demand prediction requires the procedure to be done safely and regular ongoing monitoring to occur in order to ensure adverse effects are minimised. Post-surgical counselling to minimise risky sexual behaviour is also essential (Moses, 2011). These circumstances will increase expenditure on the Indonesian health system.

\section{What is potential implication for practice?}

SR findings on 'male circumcision for prevention of heterosexual acquisition of HIV in men' conducted by Siegfried et al. (2009) bring potential implications to national and local HIV prevention guidelines in Indonesia. These include practical impacts on prevention measures, policy or regulation impacts and further research focus. Before adapting this strategy into potential areas (with highly concentrated and generalised HIV epidemics), acceptability and feasibility must be taken into consideration.

Community readiness assessment should be conducted by the national and local agencies. Several key points to be addressed include: (1) common understanding among community and leaders related to male circumcision and HIV prevention; (2) the capability of community based organisations and government bodies in delivering appropriate services; (3) identifying potential approaches to build common understanding; and (4) seeking support from leaders and community (Brownson et al., 2011). Furthermore, dissemination and implementation initiatives should be created as a foundation to support further planning strategy particularly in Papua and Bali.

Some of the findings of Siegfried et al (2009)SR are relevant to Indonesian context and promising for the reduction of $\mathrm{HIV}$ transmission. ${ }^{5}$ Male circumcision is likely however to function as an additional support program complimenting existing HIV prevention strategies. Male circumcision intervention among adult men can 
be introduced after assessment of applicability and transferability attributes are completed (eg. applicability, political environment, social and cultural acceptability, resource implications, target population educational level, available structure and skills and baseline prevalence) (Brownson et al., 2011). Further research to assess this area is essential. In parallel to this, increasing consistent condom use within commercial sex practices and reduction of clients are critical, accompanied by an educational campaign on health benefits (including HIV prevention) of male circumcision to the wider community. Significant work is required from the current situation in Indonesia before the SR findings can be integrated with national policy and regulations. The findings do, however, provide justification to immediately start promoting the health benefits of male circumcision to wider community. This can be targeted by put additional information related to male circumcision in Sexual and Reproduction Health (SRH) campaigns at schools and HIV/AIDS education programs and by increasing media publication through dissemination information strategy. Longer time is needed to promote social acceptability.

\section{CONCLUSION}

The findings from SR on 'male circumcision for prevention of heterosexual acquisition of HIV in men' are warranted to be introduced. Male circumcision can reduce HIV transmission among sexually active men by $38-66 \%$. This finding is highly relevant to Indonesian context and partially transferable because of: (1) the high prevalence of HIV/AIDS in Indonesia, ranging from concentrated and generalised HIV epidemics; (2) heterosexual contact is a major route of HIV transmission; and (3) the demographic feature of high risk population. These facts alone, however, do not sufficiently address applicability issues to justify implementation. There are three major applicability issues that should be taken into consideration: (1) HIV/AIDS epidemic and the centrality of sex workers in the transmission of HIV in Indonesia; (2) cultural and social reluctance; and (3) health system readiness. Complete community readiness assessment and a complete applicabilitytransferability attributes assessment are essential prior to adoption. This requirement mandates a new field of research agenda in Indonesia. In the current situation, the SR finding on male circumcision potentially impacts national policy and regulation particularly in disseminating information strategy. The findings provide immediate justification to start promoting the health benefits of male circumcision to the wider community.

\section{REFERENCES}

1. UNAIDS. AIDS Epidemic Update; 2009. [Retrieved 23 ${ }^{\text {rd }}$ of September 2011]. Available from http://www.unaids. org/en/media/unaids/contentassets/dataimport/pub/ report/2009/jc1700_epi_update_2009_en.pdf

2. UNAIDS. UNAIDS Report on the Global AIDS Epidemic 2010; 2011. [Retrieved 22 $2^{\text {nd }}$ of September, 2011]. Available from http://www. unaids.org/globalreport/ documents/20101123_GlobalReport_full_en.pdf

3. Wilson D. HIV Epidemiology: A review of recent trend and lesson. The World Bank: Global HIV/AIDS Program; 2006.

4. UNICEF. How widespread the HIV/AIDS epidemic is?; 2011. [Retrieved 22 ${ }^{\text {nd }}$ of September, 2011]. Available from http://www.unicef.org/aids/index_ epidemic.html

5. Siegfried N., Muller M, Deeks JJ, Volmink J. Male circumcision for prevention of heterosexual acquisition of HIV in men. Cochrane Database of Systematic Reviews 2009; 2 .

6. UNAIDS \& WHO. New Data on Male Circumcision and HIV Prevention: Policy and programme implication. WHO/UNAIDS Technical Consultation Male Circumcision and HIV Prevention: Research implications for policy and programming. Montreux, Geneva: Joint United Nations Programme on HIV/AIDS and World Health Organization; 2007.

7. Indonesian National AIDS Commission. Laporan Kementerian Kesehatan Triwulan Kedua 2011. [Retrieved on $20^{\text {th }}$ of September, 2011]. Available from http://www. aidsindonesia.or.id/laporan-kementerian-kesehatantriwulan-kedua-2011.html

8. Indonesian National AIDS Commission. Laporan Kementerian Kesehatan Triwulan Kedua 2011. [Retrieved on $20^{\text {th }}$ of September, 2011]. Available from http://www. aidsindonesia.or.id/laporan-kementerian-kesehatantriwulan-kedua-2011.html

9. Bridges JFP, Selck FW, Gray GE, McIntyre JA and Martison NA. Condom avoidance and determinants of demand for male circumcision in Johannesburg, South Africa.' Health Policy and Planning 2011; 26: 298-306.

10. Dini C. Male circumcision as strategy for HIV prevention and sexually transmitted diseases. The potential role of traditional birth attendants in neonatal male circumcision'. Ann Ist Super Sanita 2010; 46(4): 349-59.

11. Brito MO, Caso LM, Balbuena $\mathrm{H}$, and Bailey RC. Acceptability of Male Circumcision for the Prevention of HIV/AIDS in the Dominican Republic. Published online 2009 November 2. doi: 10.1371/journal. pone.0007687. http://www.ncbi.nlm.nih.gov/pmc/articles/ PMC2765614/?tool=pmcentrez

12. Kenneth M, Christopher W, Connie C, Edith NJ, Elly $\mathrm{K}$ and Jared MB. Circumcision of Male Children for Reduction of Future Risk for HIV: Acceptability among HIV Serodiscordant Couples in Kampala, Uganda. Published online 2011 July 20. doi: 10.1371/journal. pone.0022254. http://www.ncbi.nlm.nih.gov/pmc/articles/ PMC3140501/?tool=pmcentrez

13. Eaton L and Seth CK. Behavioral aspects of male circumcision for the prevention of HIV infection. Current HIV/AIDS Reports 2009; 6(4): 187-93.

14. Moses S. Male circumcision: a new approach to reducing HIV transmission. CIHR/CMAJ: Top Canadian Achievements in Health Research; 2011.

15. Brownson RC, Baker EA, Leet TL, Gillespie KN \& True WR. Evidence Based Public Health Second Edition. New York: Oxford University Press; 2011.

16. Wilson D. (2006). 'HIV Epidemiology: A review of recent trend and lesson'. Global HIV/AIDS Program. The World Bank

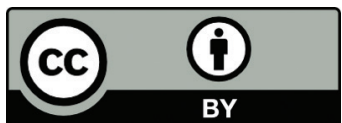

This work is licensed under a Creative Commons Attribution 